\title{
\begin{tabular}{llllllllllll} 
L & $I$ & $N$ & $G$ & $U$ & $I$ & $S$ & $T$ & $I$ & $C$ & $A$ \\
\hline
\end{tabular}
}

LETO VII.

\section{Anton Grad \\ REMARQUES \\ SUR LE STYLE INDIRECT LIBRE EN ANCIEN FRANÇAIS}

On sait que, pour rapporter les paroles ou les pensées de ses personnages, l'auteur peut se servir dé deux procédés littéraires:

$1^{0}$ du style direct (oratio recta), dans lequel les paroles (les pensées) d'une personne, introduites d'ordinaire par un verbe exprimant la parole ou ia pensée, sont rapportés avec pleine exactitude, p. ex.:

Il revint payer sa note et partit comme un voleur. Il se dit: «Maisy rapportera ma veste et j'enverrai ma secrétaire la prendre chez elle avec des fleurs.»

Le verbe introductoire peut aussi être intercalé, en incise, dans le style direct: "Maisy, se dit-il, rapportera ma veste....", ou bien il peut terminer le discours direct: "Maisy rapportera ma veste et j'enverrai ma secrétaire la prenare chez elle avec des fleurs,» se dit-il.

$2^{0}$ du style indirect (ordinaire, dépendant, subordonnée) (oratio obliqua), dans lequel l'auteur rapporte ce qui a été dit (pensé) par ses personnages en employant ses propres paroles, introduites par la conjonction que (ou un interrogatif dans les questions indirectes) et dépendant d'un verbum dicendi, etc., p. ex.:

Il revint payer sa note et partit comme un voleur. Il se dit que Maisy rapporterait sa veste et qu'il enverrait sa secrétaire la prendre chez elle avec des fleurs.

On aura remarque la transposition de la première (et c'est aussi le cas de la deuxième) personne dans la troisième, ainsi que la transposition des formés verbales: après un passé, le futur devient conditionnel (et le présent devient imparfait).

Toutefois, une troisième possibilité de rapporter les paroles (les pensées) de ses personnages est fournie à l'auteur par le procédé baptisé «le style indirect libre" par Charles Bally ${ }^{1}$ et Margueritte Lipps ${ }^{2}$ et "die erlebte Rede» par E. Lorck ${ }^{3}$, p. ex.:

Il revint payer sa note et partit comme un voleur. Maisy rapporterait sa veste et il enverrait sa secrétaire la prendre chez elle avec des fleurs.

(Sagan, Aimez-vous Brahms? ,Ch. 13; procédé très fréquent chez

Sagan!)

${ }^{1} \mathrm{Ch}$. Bally, Le style indirect libre en français moderne, dans la revue Germanisch-romanische Monatsschrift, vol. IV (1912), p. $597 \mathrm{~s}$, vol. VI (1924), p. $405 \mathrm{~s}, 456 \mathrm{~s}$.

${ }^{2}$ M. Lipps, Le style indirect libre, thèse, Paris, 1926.

${ }^{3}$ E. Lorck, Die erlebte Rede, eine sprachliche Untersuchung, Heidelberg, 1921. 
On voit que, comparé au style inđirect orđinaire (dépendant), le style indirect libre (oratio obliqua libera) est caractérisé par l'absence de la principale comportant le verbum dicendi (ou sentiendi), ainsi que de la conjonction que introduisant la subordonnée complétive du style indirect ordinaire; celle-ci devient, pour ainsi dire, indépendante (libre), bien que, dans l'emploi du temps verbal - nous reviendrons sur ce point important - le verbe en est sujet aux mêmes règles que celui de la subordonnée du discours indirect ordinaire.

Mais quel serait le but poursuivi par l'auteur ayant recours à ce procédé stylistique?

Grâce à notre tournure, l'auteur peut faire exprimer les pensées (les paroles) à ses personnages par eux-mêmes, on pourrait dire qu'il se confond avec eux, car ce n'est plus lui qui y fait son récit, qui y exprime ses propres pensées: les vrais sujets des énoncés du style indirect libre sont ses personnages.

Il est vrai que, faute de signes extérieurs du style indirect libre, très souvent seuls le contexte et la situation permettent au lecteur de deviner quel est le sujet de l'énoncé de notre tournure; c'est aussi pour cette raison que, surtout aux époques plus anciennes, de pareils passages donnent lieu à des équivoques, à une double interprétation possible: on ne sait pas très bien s'il s'agit du récit de l'auteur ou du style indirect libre, c'est-à-dire de l'expression des pensées (des paroles) des personnages mêmes (v. plus bas). Comp. encore:

Pendant les huit derniers jours, Savinien avait fait des réflexions sur l'époque actuelle. La concurrence en toute chose exige de grands travaux de qui veut une fortune. Les moyens illégauix demandent plus de tatent et de pratiques souterraines qu'une recherche à ciel ouvert. Les succès dans le monde, loin de donner une position, dévorent le temps et veulent énormément d'argent. Le nom de Portenduère, que sa mère lui disait tout-puissant, n'était rien à Paris. Son cousin le député, le comte de Portenduère, faisait petite figure au sein de la Chambre élective en présence de la pairie, de la cour, et n'avait pas trop de son crédit pour lui-même. L'amiral de Kergarouet n'existait que par sa femme. Il avait vu des orateurs, des gens venus du milieu social inférieur à la noblesse, ou de petits gentilshommes, être des personnages influents. Enfin' l'argent était le pivot, l'unique moyen, l'unique mobile d'une société que Louis XVIII avait voulu créer à l'instar de celle a'Angleterre. De la rue de la Clef à la rue Croix-des-Petits-Champs, le gentilhomme développa le résumé de ses méditations.

- Je dois, dit-il, me faire oublier pendant trois ou quatre ans, et chercher une carrière."

(Balzac, Ursule Mirouet, 1ère partie)

En vain il (sc. le colonel) parla de la sauvagerie du pays et de la difficulté pour une femme d'y voyager; elle (sc. la fille du colonel) ne craignait rien; elle aimait par-dessus tout à voyager à cheval; elle se faisait une fête de coucher au bivouac (= «je ne crains rien, disait sa fille, j'aime par-dessus 
tout à voyager à cheval, je me fais une fête de coucher au bivouac»). Elle menaçait d'aller en Asie Mineure. Bref, elle avait réponse à tout.

(Mérimée, Colomba, préambule)

"Monsieur Paul! votre maman vous appelle.» Pauline jette ces mots dans l'entrebâillement de la porte et disparaît. Poum file le long du corridor. Elle n'aurait pas l'audace de lui faire réciter sa table de multiplication, un jour de Päques? Serait.ce pour s'informer si Poum a recopié sa dictée? Il n'est pas rassuré.

(P. et V. Margueritte, Poum)

Il la retint à déjeuner. Elle attendait sa blanchisseuse, elle devait être rentreé de bonne heure. Cette réponse l'exaspéra.

(Huysmans, Marthe, p. 607)

L'officier regarda Katow:

"Morts?»

Pourquoi répondre!

«Isolez les six prisonniers les plus proches!

- Inutile, répondit Katow; c'est moi qui leur ai donné le cyanure.»

(Malraux, La Condition humaine), etc., etc.

Depuis la publication de l'ouvrage de Lorck, précédé, toutefois, de quelques études faisant déjà mention de cette tournure, ${ }^{4}$ les romanistes, ainsi que d'autres linguistes et stylistes n'ont pas cessé de porter un intérêt particulier ə̀. ce procédé líttéraire; si, tout d'abord, le style indirect libre avait semblé être une particularité caractéristique des auteurs français modernes, répandue surtout dans les textes après 1850, p. ex. chez Flaubert et Zola - bien que dès le début des recherches on ait pu en constater des exemples chez la Fontaine đéjà ${ }^{5}-$, des études spéciales pour plusieurs autres langues modernes (et aussi pour le latin) n'ont pas tardé à démontrer qu'il s'agit d'un tour connu à d'autres langues aussi, et ceci même aux époques antérieures au 190 siècle. ${ }^{6}$

${ }^{4}$ Cf., déjà en 1905, la thèse de E. Herdin, Studien über Eericht und indirekte Rede im modernen Deutsch, Uppsala; selon Herdin, Wieland aurait le premier, en Allemagne, fait usage de notre procédé littéraire; et le linguiste allemand Behaghel l'a découvert déjà en 1878.; v. sa Deutsche Syntax, III, p. $694 \mathrm{~s}$.

V, aussi O. Jéspersen, The Philosophy of Grammar, 1924, p. $290 \mathrm{~s}$; Jespersen donne au style indirect libre le nom de "represented speechir.

Th. Kalepky l'appelle "verschleierte Redeu (style voilé), dans la revue Neophilologus, XIII, p. l. s, 1927-1928.

Pour compléter la bibliographie sur notre problème, v. W. Gunther, Probleme der Redestellung. Marburg a. L., 1928.

'Comp.: Enfin, n'en pouvant plus d'effort et de douleur, Il met bas son fagot, il songe à son malheur. Quel plaisir a-t-il eu depuis qu'il est au monde? En est-il un plus pauvre en la machine ronde?

(La mort et le Bûcheron)

'Pour l'anglais, v. F. Karpf, Die erlebte Rede im älteren Englischen und in rolkstümlicher Redeweise, dans la revue Die neueren Sprachen, XXXVI, p. $571 \mathrm{~s}$; 
Voici, à titre d'illustration, quelques exemples du style indirect libre en anglais; ils appartiennent presque tous aux textes antérieurs à 1850, de manière qu'on ne saurait parler d'une influence exercée par les auteurs français modernes sur la création de notre procédé en anglais. Comme dans les exemples français, les phrases du style indirect libre des auteurs anglais doivent être attribuées, sans aucune hésitation, aux pensées ou aux paroles d'un personnage, l'auteur ne prenant pas leur contenu à son compte. Comp.:

The surgeor then advised him (sc. Joseph), if he had any worldly affairs to settle, that he would do it as soon as possible; for though he hoped he might recover, yet he thought himself obliged to acquaint him he was in great danger; and if the malign concoction of his humours should cause a suscitation of his fever, he might soon grow delirious, and incapable to make his will. Joseph answered, that...

(Fielding, Joseph Andrews, I, Ch. 13)

I lamented with unfeigned sorrow his (sc. Jack Rattlin's) misfortune, which he bore with heroic courage, observing that every shot had its commission. It was well it did not take him in the head, or, if it had, what then? he should have died bravely fighting for his king and country: death was a debt which every man owed, and must pay; and that now as well as another time. I was much pleased and edified with the maxims of this sea philosopher, who endured the amputation of his left hand without shrinking.

(Smollett, Roderick Random, Ch. 32)

Notre tournure est extrêmement fréquente dans les romans de Jane Austen (morte en 1817) qui la cultive avec une faveur vraiment surprenante, comp.:

Mrs. Bennet invited him (sc. Mr. Bingley) to dine with them; but, with many expressions of concern, he confessed himself engaged elsewhere.

"Next time you call," said she, "I hope we shall be more lucky."

He should be particularly happy at any time, etc., etc., and if she would give him leave, would take an early opportunity of waiting on them.

"Can you come to-morrow?"

Yes, he had no engagement at all for to-morrow; and' her invitation was accepted with alacrity.

(Jane Austen, Pride and Prejudice, Ch. 55)

I asked if Georgiana would accompany her. - Of course not. Georgiana and she (sc. Eliza = Georgiana's sister) had nothing in common; they never

O. Funke, Die erlebte Rede bei Galsworthy, dans la revue Englische Studien, LIV; p. $450 \mathrm{~s}$; L. Glauser, Die erlebte Rede im englischen Roman des 19. Jahrhunderts, Bern, 1948.

Pour l'espagnol, v. Fr. Todemann, Die erlebté Rede im Spanischen, dans la revue Romanische Forschungen, XLIV, p. $103 \mathrm{~s}$.

Pour l'italien, v. Spitzer, dans la revue Germanisch-rómanische Monatsschrift, 1921, p. $59 \mathrm{~s} ;$ G. Herzeg, Lo stile indiretto libero in italiano, Firenze, 1963.

Pour le latin, v. J. Bayet, Le style indirect libre en latin, dans la Revue de philologie, de littérature et d'histoire anciennes, V (1931), VI (1932). 
had had. She could not be burdened with her society for any consideration. Georgiana should take her own course; and she Eliza, would take hers.

(Charlotte Brontë, Jane Eyre, Ch. 21)

All that the Rector could say could not bring Helen to feel any indignation or particular unhappiness, except that the boy (sc. her son) should be unhappy. What was this degree that they made such an outcry about, and what good would it do Pen (sc. her son)? Why did Doctor Portman and his uncle insist upon sending the boy to a place where there was so much temptation to be risked, and so little good to be won? Why didn't they leave him at home with his mother? As for his debts, of course, they must be paid; his debts! - wasn't his father's money all his, and hadn't he a right to spend it? In this way the widow met the virtuous Doctor...

(Thackeray, Pendennis, p. 238)

Un exemple très intéressant du style indirect libre est fourni par Dickens: tout un dialogue (devant le tribunal) est rapporté par l'auteur au moyen de notre procédé stylistique, comp.:

... he (sc. the witness) would have modestly withdrawn himself, but that the wigged gentleman... begged to ask him a few questions. The wigged gentleman sitting opposite, still looking at the ceiling of the court.

Had he ever been a spy himself? No, he scorned the base insinuation. What did he live upon? His property. Where was his property? He didn't precisely remember where it was. What was it? No business of anybody's. Had he inherited it? Yes, he had. From whom? Distant relatives. Very distant? Rather. Ever been in prison? Certainly not. Never in a debtor's prison? Didn't see what that had to do with it. Never in a debtor's prison? - Come, once again. Never? Yes. How many times? Two or three times. Not five or six? Perhaps. Of what profession? Gentleman. Ever been kicked? Might have been. Frequently? No. Ever kicked downstairs? Decidedly not; once received a kick on the top of the staircase and fell downstairs of his own accord. Kicked on that occasion for cheating at dice? Something to that effect was said by the intoxicated liar who committed the assault, but it was not true...

(Dickens, A Tale of Two Cities, Book II, Ch. 3)

Comme đéjà mentionné ci-dessus, E. Herdin, dans sa thèse de 1905, constate notre tournure chez des auteurs allemands, comme Wieland, Wildenbruch, Tovote, Fontane, Otto Ludwig, Alexander Baron Roberts, etc.; nous ne voulons citer que deux de ses exemples:

Er wollte sie malen... Aber sie weigerte es ihm... Nein, sie wollte es nicht. Sie konnte nicht still sitzen. Sie wollte sich ihr Gesicht nicht steh̆len lassen. Siè ging ja auch nicht zum Photographen... Er durfte ihr deshalb nicht böse sein. - Und er verzichtete auf den Wunsch.

(Tovote, Im Liebesrausch, p. 256)

Holk fühlte sich als er (den Brief seiner Frau) gelesen, einer gewissen Rührseligkeit hingegeben, Es war so viel Liebes in dem Briefe, dass er alte 
Zeiten und altes Glìck wieder heraufsteigen fühlte. Sie war doch die beste. Was bedeutete daneben die schöne Brigitte? ja, was bedeutete daneben selbst. Ebba? ...

(Fontane, Unwiederbringlich, p. 183)

Lorck, o. c., donne l'exemple suivant de Thomas Mann:

Der Konsul ging, die Hände auf dem Rücken umher und bewegte nervös die Schultern. Er hatte keine Zeit. Er war bei Goot ïberhäuft. Sie (sc. sa soeur à qui ses paroles sont adressées) sollte sich gedulden und sich gefälligst noch fünfzig mal besinnen!

(Th, Mann, Buddenbrooks, I, p. $562 / 1901 /$ )

Il serait superflu de donner aussi des exemples italiens, espagnols, etc., mais avant d'aborder nos recherches concernant le style indirect libre en ancien français, un exemple emprunté au latin ne serait peut-être pas sans intérêt:

Tulit Caesar grauiter. Litterae Capuam ad Pompeium uolare dicebantur: Inimici erant equitibus qui Curioni stantes plauserant, hostes omnibus. Rosciae legi, etiam frumentariae minitabantur. Sane res erat perturbata. Equidem malueram quod erat susceptum ab illis silentio transiri, sed uereor ne non liceat.

(Cic., ad Att., II, 19, 3-4, cité par Bayet, o. c., V, p. 330)

En ancien français aussi, notre tournure est plus fréquente que ne le laissent supposer 'les études publiées par $M$. Lipps et Gertraud Lerch, ${ }^{8}$ et la constatation de $M$. Lipps que «en vieux français l'indirect libre à l'état de figure n'existe qu'à titre exceptionnel et se trouve employé dans des circonstances spéciales" (o. c., p. $127 \mathrm{~s}$ ) ne' pourrait - nous ne tarderons pas à le voir - être acceptée qu'avec une certaine réserve.

Voici les passages cités déjà soit pär M. Lipps soit par G. Lerch comme contenant incontestablement notre procédé stylistique:

E dist' (sc. Blancadrins) al rei (sc. Marsilies):

Mandez Carlun, a l'orguillus e al fier,

Fedeilz servises e mult granz amistez.

Vos li durrez urs e leons e chenz,

Set cenz camelz e mil hosturs muers,

D'or e d'àrgent. IIII. C. muls cargez,

Cinquante carre qu'en ferat carier:

Ben en purrat luer ses soldeiers.

En ceste tere ad asez osteiet:

En France, ad Ais, s'en deit ben repairer.

Vos le sivrez a la feste seint Michel,

Si recevrez la lei de chrestiens,

Serez ses hom par honur e par ben.

Roland, v. 28-39

\footnotetext{
${ }^{7}$ Pour l'allemand, v. aussi O. Walzel, Gehalt und Gestalt im Kunstwerk des Dichters, 1929 , p. 240 ., p. 380 s.

s Gertraud Lerch, Die uneigentlich direkte Rede, dans Idealistische Neuphilo logie, Festschrift für Karl Vossler, Heidelberg 1922, p. $107 \mathrm{~s}$.
} 
Blancadrin ne prend pas son discours au sérieux: il ne fait que suggérer au roi Marsile les paroles qu'il lui faut transmettre à Charlemagne, et c'est pourquoi aussi Gautier, dans sa traduction du passage, y introduit l'incise "direz-vous»: "Vous l'y suivrez - direz-vous - à la fête de saint Michel; Et $l a \dot{a}$, vous vous convertirez à la foi chrétienne, Vous serez son homme en tout bien, tout honneur., Ce passage représenterait donc peut-être le plus ancien exemple de notre tournure en français.

Il faut probablement voir un cas semblable dans le passage suivant où notre tour exprime aussi un ordre:

(La demoiselle /sc. Lunette/ dit à la maîtresse du château) Sour moi laissiez ceste besoigne,

et endementres manderoiz
vos genz et si demanderoiz
consoil del roi qui doit venir.
Por la costume maintenir
de vostre fontaine defendre
vos covendroit bon conseil prendre.
E il n'i avra ja si baut
qui s'ost vanter que il i aut.

(Chr. de Troyes, Le Chevalier au lion, v. 437-447)

La situation nous fait comprendre que les vers $445-447$ contiennent une suggestion faite par Lunette à sa maîtresse de ce que cette dernière a à dire, et non à faire, et, en effet, dans leur Chrestomathie du moyen âge, 1917, G. Paris et $\mathrm{E}$. Langlois traduisent: "Dites-leur qu'il convient d'aviser à défendre votre fontaine suivant la coutumes"

M. Lipps cite aussi l'exemple suivant qui, en effet, suggère l'idée d'une sorte de style indirect libre:

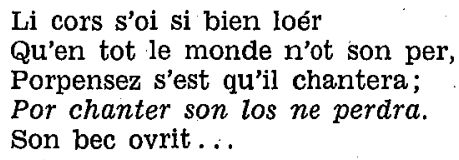

(Le goupil et le corbeau, Clédat, Chrestomathie, p. 241)

Le vers: Porpensez s'est qu'il chantera prépare le vers suivant: Por chanter son los ne perdra, qui exprime la pensée du corbeau à l'aide de notre tournure.

A ces exemples assez sûrs du style indirect libre ajoutons-en quelques-uns que nous avons relevés au cours de nos lectures des anciens textes et qui, croyons-nous, ne prêtent à aucune ambiguïté: c'est encore la situation ou le contexte qui suggère l'idée de l'indirect libre, car les phrases en question ne pourraient pas être attribuées à l'auteur, mais à ses personnages. Comp.:

Et cil au Dragon s'apareille, Qui molt durement se merveille Qui cil est qui tant a hardie La char que la cloche a bondie 
Si fort et de si grant vertu:

C'est des barons le roi Artu

Qui chi vient mostrer son esfort,

Mais ja morra de laide mort.

En son tref failt un drap estendre,

Armer se fait sans plus atendre.

(Gerb. de Montr., Perceval, 9475-84)

La suite des événements, elle aussi, va montrer que les vers $9480-82$ ne représentent pas le récit direćt, l'opinion de l'auteur, mais la pensée, le soupçon et la menace du chevalier au dragon.

Voici un autre passage, emprunté au même texte, qui semble, avec beaucoup de probabilité, représenter aussi notre tournure:

Quant Perchevaus ot la raison

Et la vois de Gavain oi,

Molt durement s'en esbahi,

Car au parler Gavain li samble.

Mais de corrous et d'ire tramble

De ce qu'au col voit l'estrument,

Si se merveille durement,

Se che est il, por quel affaire

Il se voloit menestreus faire.

Se ce est il bien le sara,

Que son non li demandera:

Bien le sara ja au parler,

C'onques son non ne volt celer

A nului qui li demandast

Por nule rien que il doutast.

Lors li a dit: "S'il vous plaist, sire,

Vostre non vous estuet ainz dire

Et puis vous redirai le mien.s

(ibid:, 4648-65)

Les vers en italique représentent, selon nous, notre procédé stylistique, exprimant les pensées, la décision de Perceval.

Atant ad pris (sc. la femme de Gui) une espee,

De l'eschalberc l'ad sachee;

Puis ad dit.qu'ele se ocirad,

Quant sun seignur perdu ad.

Endré sun quor l'espee mis ad,

Quant ele dunc se purpensad

Qu'ele feseit folie grant:

Dune ert ele enceinte d'enfant?

Oscire pas ne se purreit,

Que l'enfant morir n'estovereit.

S'ele se socie en tel manere,

Quant le savera le cunte, sun pere,

$E$ sa mere $e$ ses amis

E la gent de tut le pais,

Tost quidereient que sun seignur

Oscise l'avreit par folur

$E$ pur ço fui s'en serreit;

Alterement pur"veir s'oscireit.

Unques la nuit ne reposa,

Mais'sun grant duel demena.

(Gui de Warewic, 7753-72) 
Dans le passage ci-dessus, les vers en italique rapportent la luttte intérieure de la jeune femme, lutte causée par la perte (supposée) de son mari et les raisons qui l'empêchent de se suiciđer; et ces sentiments, ces pensées sont révélés au iecteur par le personnage (la jeune femme) lui-même, et non point par l'auteur comme le prouve aussi l'emploi du conditionnel présent l'auteur y aurait recouru au conditionnel passé: on a donc affaire à l'indirect libre.

Comme le cas précédent, l'exemple suivant aussi nous fait voir une question rapportée au moyen de notre tour:

Li tiers dient que trop tardant

Va li quens qui ne se delivre:

Pour quoi la (sc. sa tante) lesse il tant vivre?

Face la a cevaus detraire

Et tous lez membres du cors traire,

Et puis la lesse an chienz mengier.

Ainsi s'en pourra bien vengier.

(Le Roman du Comte d'Anjou, 7790-96)

Les menaces, ainsi que les désirs de faire subir une mort terrible à la tante traîtresse ne peuvent être attribués à l'auteur, mais à ses personnages.

Dans les lais de Marie de France, il y a aussi des passages qui font penser a) notre procédé:

Femme voleient qu'il (sc. Guigemar) preisist

Mes il del tut les escundist:

Ja ne prendra femme a nul jor,

Ne pur aveir ne pur amur,

S'ele ne peust despleier

$\mathrm{Sa}$ chemise sans depescer.

(Marie de France, Lais, Guigemar, 645-50)

Puis avient si qu'a une feiz

Qu'a s'amie vient li danzeus,

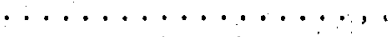

Sa pleinte li mustrat e dist;

Anguissusement li requist

Que s'en alast od lui:

Ne poeit mes suffrir l'enui.

S'a sun pere la demandot,

Il saveit bien que tant l'amot

Que pas ne li vodreit doner,

Se il ne la peust porter

Entre ses bras en sum le munt.

(ibid., Les dous amanz, 70-81)

C'est encore la situation et le contexte qui parlent en faveur de l'hypothèse que, dans les deux cas ci-dessus, nous avons affaire au style indiredt libre: on rapporte les paroles d'un personnage à l'aide de cette tournure, les phrases en question pourraient à peine être attribuées à l'auteur.

Voici encore trois exemples qui, selon nous, représenteraient aussi notre tournure, car l'interprétation des passages en italique semble se prêter plus en faveur de l'indirect libre que du récit du poète, la ponctuation du texte, 
elle aussi (si elle est exacte), prouvant une pause là où le discours indirect subordonné continue sans conjonction:

Li preudom, qui molt fu cortois,

Pria de remanoir un mois

Le vallet qui dalez lui sist.

Un an tot plain, se il volsist,

Le retenist molt volentiers,

$\mathrm{Si}$ apresist endementiers

Tels choses, s'eles $l i$ pleüssent,

Qu'au besoing mestier li eïssent.

(Le Roman de Perceval, éd. W. Roach, 1959, v. 1571-78)

Qant Brichemers l'a entendu, tornez s'en est col estandu, a Isengrin dist en l'oreille que li rois forment se mervoille

qu'en ne puet pes entre els. II. mestre

ne por doner ne por promestre:

face le bien, preigne droiture

de Renart, por sa forfaiture.

et por ce que il sor li mist

que a sa fame force fist.

(Le Roman de Renart, VIII, v. 8261-70)

(Les vers $8267-70$ comprennent les parolès, les conseils de Brichemer à Isengrin.)
Lors s'avise, que il sera
Pres du tournoi et bien verra
Li quel seront en leur desus.
Lors maintenant montera sus
Le destrier, qui tout blanc sera
Des armes qu'entour li avra,
Et a ceux, qui plus peu seront,
Et qui plus de meschief aront,
Devers euls se vorra il mettre,
Et son secours tout leur promettre.
Si con le devisa il fist;

(Le Roma de la Dame à la Licorne, v. 965-75)

(C'est un personnage de l'auteur, et non l'auteur lui-même, qui fait la réflexion exprimée par les vers en italique.)

Dans l'exemple suivant aussi nous croyons pouvoir voir, dans les vers en italique, notre tournure: les paroles, les menaces qui y sont exprimées doivent être attribuées plutôt aux barons qu'au narrateur:

(Li rois)...

Lors dit (sc. à ses barons) que li blasmes est lor

De son tribol et de sa guerre,

Car par aus bailla il sa terre

Et mist an la main au felon

Qui pires est de Guenelon.

N'i un seul qui bien n'otroit

Que li rois a reison et droit,

Car ce li conseilllierent il; 
Mes il an iert mis an essil, Et sache bien de verité Que an chastel ne an cité Ne porra garantir son cors Qu'a force ne l'an traie fors. Ensi le roi tuit aseurent Et afient formant et jurent Que le traitor li randront Ou ja mes terre ne tandront.

(Ch. de Troie, Clinés, v. 1072-76)

Les verbes au futur du passage en question résultent de la perspective des personnages du narrateur plutôt que de celle du conteur; comme le dit Vossler,' qui donne à ce futur le nom de futurum advocaticum, le conteur "s'il ne considère pas le fait de son héros comme tout à fait sien, l'envisage de toute façon comme nôtre, en quelque sorte..."

Voici encore quelques exemples de ce futur dans des passages qui suggèrent l'idée d'une sorte de style indirect libre:

tote nuit veillier li estuet (sc. à Enide),

mes ainz le jor, se ele puet

et ses sires la voelle croirre,

avront si atorné lor oirre

que por neant vanra li cuens,

que ja n'iert soe, ne il suens.

Erec dormi, molt longuement, tote la nuit...

(Chr. de Troie, Erec, v. 3446-52)

Uns golpis vint, qui l'espia (sc. le corbeau)

Del formage ot grant desirier

Que il en puist sa part mangier;

Par engin voldra essaier

Se le corp porra engeignier

(Le goupil et le corbeau, Clédat, Chrestomathie, p. 241)

Voitle Escorfaus, a poi de deul ne fent, S'il le puet faire, il le fera dolent;

(Bueve de Hantone, v. 9273-74)

Prise li (sc. au comte) est grant volempté

De soy vengier de la contesse

S'antain, la mortel traitresse,

Leur (sc. de ses barons) conseil en voudra avoir

Et au roy le fera savoir,

Qui son plesir li rescrira,

Et sagement, s'il puet, ira

Avent en si grosse besoingne.

Lez barons vienent sanz esloigne

Et tuit si homme sanz respit;

(Le Roman du Comte d'Anjou, v. 6794-6807)

${ }^{9} \mathrm{~K}$. Vossler, Langue et Culture de la France, traduction d'Alphonse Juilland, Paris, 1953, p. 62. 


\section{Anton Ġrad}

Dunques"pense (sc. Gui) que faire peust;

Mais ore se voldra il taisir,

A nul ne se voldra descovrir.

As puceles s'en est alé,

(Gui de Warewic, v. 228-31)

Quant Gui vit Heralt trebucher

$\mathbf{E}$ aloigné de sun destrer,

Pur poi que de doel n'araga;

S'il pot, ja le vengera.

(ibid., v. 1327-30)

Purpeńsé ad (sujet $=$ Morgadur) en sun corage

Que Gui frat aler en un message

Al soldan, qui tant es fier;

S'il va, mes n'iert del repairer.

(ibid., v. $3685-88$ )

mout se demente, mout se duet (sc. Brun, l'ours)

encor tenra Renart, s'il puet,

qui tot ce li a porchacié.

(Le Roman de Renart, VII b, 6772-74)

Il pense qu'il l'estuet pener

De fuir au plus qu'il porra;

S'il puet dusc'au recet corra,

C'une forest devant lui voit:

S'ançois d'aus venir i pooit,

A tos jors mais perdu l'aroient;

Ja mais noveles n'en saroient.

(Guillaume d'Angleterre, v. 1668-74)

Quant l'ot li rois, molt l'an est grief,

et jure assez plus que son chief

que cil qui l'(sc. Lancelot) ont mort an morront;

ja desfandre se s'an porront

'et, s'il les puet tenir ou prandre,

ja n'i avra mes que del pandre

ou del ardoir ou del noier.

(Chr. dr. Tr., Le Chevalier de la Charrette, v. 4143-49), etc., etc.)

Les passages, comportant ce futurum advocaticum et très fréquents dans la vieille langue, sont nés des pensées du héros plutôt que de celles du conteur; celui-ci s'identifie à lui, il est pour aịnsi dire uemporté dans le tourbillon des sentiments, des passions et des décisions exprimés par ses personnages" (G. Lerch, o. c., p. 108); lui qui cependant connaît la suite des événements et la fin de l'histoire qu'il raconte, semble l'oublier comme le prouve aussi l'expression s'il puet, si fréquente dans ces tournures et qui, logiquement, ne pourrait être attribuée qu'au héros qui; lui, à ce moment-là, ignore les événements à venir.

Comme déjà mentionné par M. Lipps. o. c., p. 119, l'ancien français pouvait arriver au style indirect libre - ou plutôt à un style ressemblant, par sa structure, en partie à l'indirect libre - aussi par la voie effectuée par l'omission de la conjonction que dans le style indirect "classique $\dot{c}=$ sub- 
ordonné); Bally déjà, sans affirmer explicitement que le style indirect libre ne serait qu'une modification mécanique du style indirect subordonné, a constaté le même fait dans son article cité ci-dessus où il dit textuellement: "Une première dérogation à la règle du style indirect pur consiste à introduire la ou les premières propositions par des conjonctions et à donner aux autres une forme non conjonctionnelle»; selon lui, la phrase de Cherbuliez:

Mlle Ferray mit fin à ce débat en déclarant qu'elle prenait tout sur elle, qu'elle assumait toutes les responsabilités, qu'elle se chargeait de toutes les explications, bref, qu'elle se faisait fort d'obtenir le pardon de Meg. (Miss Rovel, p. 33)

pourrait être modifiée comme suit:

Mlle Ferray mit fin à ce débat en déclarant qu'elle prenait tout sur elle: elle assumait toutes les responsabilités;" elle se chargeait de toutes les explications; bref, elle se faisait fort d'obtenir le pardon de Meg. ${ }^{10}$

Ces conclusions de Bally n'ont pas rencontré l'approbation de Lorck pour qui surtout l'omission de la conjonction est incompréhensible et inadmissible.

Or, s'il est, en effet, difficile de compter avec une pareille omission dans la langue littéraire moderne (à règles syntaxiques bien fixées et imposées aux écrivains), de telles tournures peuvent être largement prouvées pour l'ancien français, langue dans laquelle la structure logique et grammaticale de la phrase n'est pas encore fixée et est loin d'être claire dans tous les cas. Nous voudrions attirer l'attention des syntacticiens sur de telles constructions très fréquentes particulièrement après le verbe jurer jouant le rôle du verbum dicendi introductoire; et - last not least - même la première proposition de l'indirect y montre une forme non conjonctionnelle, un cas donc où il ne s'agit pas d'éviter une répétition de la conjonction, répétition évitée pour raisons d'ordre euphonique qui, selon M. Lipps, serait la cause principale de son omission. Comp.:

Elle en a juré saint Guillain

Ja li hoir qui de li vendra

La terre au conte ne tendra:

Bien i savra remede mectre

Et molt s'en voudra entremétre,

Soit tort, soit droit, en quelque guise.

(Le Roman du Comte d'Anjou, v. 3052-57)

Dos de Mainche...

Dieu a juré, le roi de paradis,

Cil qui l' (sc. son neveu) a mort ne s'en ira ja vis.

(Bueve de Hantone, v. 4621-23)

${ }^{10}$ Bally, o. c., p. 553.

"Lorck, o. c., p. 24: "Ihre (sc. der erlebten Rede) Entstehung versuchte Bally... rein grammatisch aus Vorgängen im Schosse der Sprache zu erklären. Der mstyle indirect librew sei eine blosse neuere Abart der mforme classique du discours indirectu und habe sich auf dem Wege: il disait qu'il était malade, il disait: il était malade, il était malade (disait-il) herausgestaltet. Unbegreiflich bei diesem. Prozesse ist vor allem der Ausfall der Konjunktion que. Bally begrïndet ihn durch die neuzeitliche Neigung, die Sätze zu koordinieren $\therefore$... 
Dame Hersant i est venue, Isangrin est remés en mue; novelement laissié l'avoit par un mehaing ( = blessure) que il avoit; Dieu jure et Sainte Patenostre ja mes ne gerra a sa coste: qu'a l'en a faire d'ome en chanbres, puis que il n'a trestoz ses manbres? mais voit a Dieu, si se porchat, droiz est que tot li monz le chat; por tant s'en est de li tornee.

(Le Roman de Renart, v. 2934-44)

Si con l'amirant jure sa foi et sa pansee, De ci que a Laon ert s'ensengne criee, $A$ seint Denis en France sa teste coronnee, Puis vendra a Paris, la grant cité loee; S'il puet prendre Aymeri, la teste avra copee; Dame Hermenjart sera as escuiers livree.

(Le Siège de Barbastre, v. 32-37), etc.

Les cas de ia suppression de la conjonction que dans la deuxième (troisième, etc.) proposition dépendante ,motivée probablement aussi par la distance séparant le verbe jurer de la proposition (des propositions) complément ,sont bien plus nombreux encore: ces propositions compléments prennent pour ainsi dire une forme indépendante et suggèrent à juste titre l'idée du style indirect libre. Comp. encore:

... et ele s'en ala

Droit a Hanstone, dont li dus le doa,

Son fil Beuvon ensamble lui mena

Et les omages reciut de ciaus de la;

Del duc qu'est mors molt s'en esleecha,

Dieu a juré qui le monde forma

Que ja ses fieus quinze jors ne vivra;

Se plus ne puet, a ses mains l'ochira,

Puis prendra Do, qui en ses bras gerra,

Ja le viellart ne li resamblera.

(Bueve de Hantone, v. 125-133; éd. p. A. Stimming, 1911)

Et Danemons Mahomet en jura,

Que ensi Bueves ja ne s'en tornera,

Il l'ochira, ja mais ne mengera;

Cil (sc. Askin, tué par Bueve) eret ses roces, et il le vengera.

(ibid., v. 1275-78); ibid. 220-31, 360-71

Iluec se sont li doi roi acordé,

Le siege jurent a le bonne cité:

N'en partiront por vent ne por oré

Desc'a cele eure que seront afamé;

Se les (sc. les Francais) pueent prendre par vive poosté, Pendu seront et au vent encroé.

(Huon de Bordeaux, v. 8311-16) 
Puis me fist sur seinz jurer

Qu'en cest message deusse aler

E a lui puis repaier;

Se nul de ces ne peusse amener,

A grant dolur me freit morir,

Nule rien ne m'én purreit garir,

Ensemble od mei mes quinze fiz;

(Gui de Warewic, v. 8125-31), etc.

On retrouve l'omission de la conjonction que aussi après d'autres verbes comme p. ex. dire, mander, faire savoir, sentir, etc.:

Cil s'esveille pour la grant noise,

Et il li dient qu'il s'en voise:

Trop a dormi, trop se delaie.

(Le R. du Comte d'Anjou, v. 3713-15)

Celes que en la chambre esteient

La (sc. la mère qui veut faire mourir une de ses filles jumelles) confortouent e diseient

Que eles nel suffereient pas;

De humme ocire n'est pas gas.

(Marie de France, Lais, Le Freisne, v. 95-98)

Renart dist que mauz ne demande;

tres bien $i$ ert et bien fera

quant que la cort esgardera

(Le Roman de Renart, VII b, v. 6772-74)

Si leur fait on a tous savoir

Que dimenche y erent les noces;

Tant $y$ avra d'abbez a croces,

Et clers, et evesques mittrés,

Et chevaliers logiez en trez

Aux champs, pour eulx moins encombrer,

Que nuls n'yert sages du nombrer.

S'en est chascuns garniz par ban.

(Galeran de Bretagne, v. 6694-6701)

A la roine a on cest plait conté,

Con li mes Carle avoit al roi parlé.

El prent un mes, si a Balant mandé

Qu'il li amaint en mi liu de son tré;

Veoir le volt; Balans l'a creanté.'

( La Chanson d'Aspremont, v. 946-48), etc.

Ço sent Rollant la veüe ad perdue,

(La Chanson de Roland, v. 2297)

On voit donc que, pour exprimer la subordination d'une proposition complétive, l'ancienne langue, plus primitive et n'observant pas encore strictement les règles d'hypotaxe, recourt très souvent à la simple parataxe. 
Faut-il chercher l'origine - ou une des origines - du style indirect libre en (ancien) français dans des tournures de ce type (comme le feraient supposer les explications de Bally, à savoir dans des propositions complétives devenues indépendantes grâce à l'omission de la conjonction, et, peu à peu, pour aboutir à sa forme définitive, grâce à l'omission du verbum dicendi introductoire, signe en premier lieu caractéristique du style indirect libre dans la langue moderne?

Sans nier la possibilité d'un tel développement, nous croyons pouvoir proposer une autre explication qui, jusqu'ici, n'a pas encore été prise en considération.

On a assez tardé, croyons-nous, à constater qu'il y a, dès les premiers textes en ancien français, une tournure qui, elle; forme pour ainsi dire le pont entre le style indirect ordinaire ("classique») et le style indirect libre. Nous pensons à des tournures du type suivant:

Un Sarrazin i out de Sarraguce;

Fiance prist de Guenelun le cunte, Par amistiét l'en baisat en la buche, Si l'en dunat sun helme et s'escarbuncle Tere Maior, co dit, metrat a hunte, A l'emperere si toldrat la curone.

Siet el ceval qu'il cleimet Barbamusche, (La Chanson de Roland, v. 1483-91)

Fremaus presentent a chascun;

Me sire Gavains en prist un, Si l'a tantost a son col mis, Mais Tristrans n'a pas le sien pris:

Fremail, ce dist, ne meteroit, Ne en son doit anel n'aroit Dusqu'al terme qu'il avoit mis $A$ tele $a$ en alcun pä̈s.

(Gerb. de Montr., Perceval, v. 3671-78)

Locrin en ad Hestrild amee

E a garder l'ad commandee. A muiller, ço dist, la prendra, $\mathrm{Ja}$ altre feme nen avra.

Ne volt al pais arester, Ainz passera, ceo dit, la mer. Al reaume de Loengre ira, Une piece se deduira.

Sa femme en la terre larra, A ses hummes cumandera, Que il la gardent lëaument $E$ tuit si ami ensement.

A cel cunseil s'est arestez, Si s'est richement afumez. 
La prieuse li (sc. à Fresne) voulst chercher

Sergent ou garson ou compaigne:

Fresne avec li mener ne daigne;

N'en a, ce dit, talent ne cure

Car la terre est moult bien sëure;

Serjans avra a voulenté, Qu'ell'a de l'avoir a plenté

Pour faire despens vespre et main.

Escourgiez tient en sa main

(Galeran de Bretagne, v. 4102-10)

Il va de soi qu'on trouve aussi des exemples avec le verbe intercalé dire au pluriel, ainsi que des exemples avec un verbe exprimant la pensée. Comp.:

Et cil qui voient la besoigne,

De' riens nule nel contredient. -

Por quoi? - Il vuelent mieuz, ce dient,

Assez vivre et avoir ades

Que morir d'armes desconfés.

(Meraugis de Portlesguez, v. 3378-82)

...et quant cil de l'ost voient

Que il s'en vet, s'il nel convoient,

Ce dient, ja mes n'avront joie.

(ibid., v. 4155-57)

S'espuse li unt amenee;

Sa mere est od li alee,

De la meschine aveit poirr,

Vers ki ses sires ot tel amur

Que a sa fille mal tenist

Vers sun seignur, s'ele poïst.

De sa meisun la getera,

A sun gendre cunseilera

Qu'a un produme la marit,

Si s'en deliverat, ce quit.

(Marie de France, Le Freisne; v. 363-72)

Par devant lui trespasser voit

Le graal trestot descovert,

Ne ne set pas cui l'en en sert

Et si le volroit il savoir

Mais il le demandera voir,

Ce dist et pense, ains qu'il s'en tort,

A un des vallés de la cort;

Mais jusqu'al matin atendra,

Que al seignor congié prendra

Et a toute l'autre maisnie.

Einsi la chose a respitie,

(Le Roman de Perceval, v. $3300-3310$ ), etc.

On aura remarqué que, dans les tournures ci-dessus, il s'agit d'une forme particulière du style indirect simple qui présente le verbum dicendi intercalé dans le discours même (mais il peut aussi terminer le discours indirect), une forme donc qui a son parallèle dans le discours direct où - nous l'avons vu au début de cet article - le verbum dicendi peut aussi etre intercalé dans le discours direct; toutefois, en ancien français, les deux incises diffërent, par 
leur structure, l'une de l'autre: tandis que, dans le discours direct, le verbe de l'incise, dépourvu du pronom démonstratif neutre $c e$, se fait suivre (au singulier) du pronom sujet (il, elle), celui du discours indirect est régulièrement précédé du démonstratif ce et dépourvu du pronom sujet. Comp. encore:

"Seignurs," dist il, "mult malement nos vait!"

(La Chanson de Roland, v. 2106)

Ne leserat, ço dit, que n’i parolt:

(ibid., v. 1206) (12)

La même forme de l'inđirect à l'incise intercalée peut être constatée aussi pour le moyen anglais, comp.:

As he which come was to manne,

Unto the King of Crete thanne,

Preiende that he wolde him make

A kniht and pouer with him take,

For lengere wolde he noght beleve,

He seith, both preith the king of leve

To gon and cleyme his heritage

And vengen him of thilke oultrage

Which was unto his fader do.

The king assenteth wel therto,

(Gower, Confessio Amantis, The Tale of Orestes, v. 1967-76)

Et voici déjà un exemple latin, cité par Bayet, o. c., p. 21:

Ego tantumdem dabo quantum ille poposcerit? Poscet omne quantum exarauero! - Quid omne? plus immo, inquit, si uolet (discours direct: "Plus immo, si uolam») - Quid tum? quid censes? - Aut dabis aut contra edictum fecisse damnabere.

(Cic., in Verr., III, 10, 25)

Quelle serait l'origine de cette forme du style indirect? Elle provient, à notre avis, de la forme ordinaire de l'indirect du type suivant qui, lui aussi, peut être constaté dans la vieille langue:

Coo dit li reis que sa guere out finee

(La Chanson de Roland. v. 705)

Ço dient ore tuit cil de la cité

$\mathrm{E}$ tut l'altre barnage qu'il i out asemblé:

Que cist pelerin n'est pas home mortel,

Ainz est angle que Deus tramist del ciel

Pur ocire cest diable suiduiur,

Deu ne volt qu'il regne ultre cest jur;

Se cest pelerin ne fust home faé,

Ja l'eust li dux mort e detrenché;

N'est home el mund, qui ore seit né,

Qui ses granz colps eust tant enduré.

(Gui de Warewic, vi, 10101-11)

${ }^{12}$ Sur ce problème, v. notre article: Contribution à la syntaxe des pronoms personnels sujets en ancien français, vol. V, p. $4 \mathrm{ss,} \mathrm{de} \mathrm{cette} \mathrm{revue.}$ 
Ço dient qu'il est de Afrique né;

Cil est en bataille plus doté

Que ne sunt cent chevalers armé;

Colebrant, ço dient, est apelé,

Tant fer vassal ne fu unc né.

(ibid., v. 10808-12), etc.

On voit que ce type de l'indirect ordinaire est représenté par une principale comportant le verbe dire (ou un autre verbe de parole ou de pensée) a la $3 \mathrm{e}$ personne (singulier ou pluriel), généralement au présent ou au passé simple, précédé du démonstratif neutre ço $(c e o, c e)$ et suivi de la subordonnée complétive introduite par la conjonction que. Cet ordre des propositions (objectif, logique), à savoir la principale, qui nous fait savoir celui qui rapporte les faits de la complétive, en tête et suivie de la subordonnée, s'adapte et correspond bien au récit normal, naturel, objectif de l'auteur: celui-ci n'est pas pressé de rapporter les faits, les événements (ou l'événement) contenus dans la complétive, il se contente de les annoncer au début de la phrase en y employant le démonstratif ce, antécédent de la complétive.

Or, il se peut que, pour des raisons d'ordre affectif, le récit du conteur prenne une autre tournure: l'auteur, impressionné par les faits rapportés dans la subordonnee, a, pour ainsi dire, hâte de les apprendre au public (au lecteur) - il omet, naturellement, la conjonction que - en mettant, impulsivement, la complétive en tête de la phrase, et ce n'est qu'au cours ou à la fin $d u$ récit de ces faits qu'il nous en révèle le vrai rapporteur (c'est-à-đire un ou plusieurs de ses personnages) à l'aide de l'incise ce di(s)t, ce dient, etc.; cette incise n'est que la principale transposée de l'indirect ordinaire, qui, même à sa nouvelle place, continue d'exercer la même influence sur l'emploi des temps verbaux dans le style indirect libre que dans l'indirect dépendant (ordinaire).

Voici encore quelques-uns des nombreux exemples de ce procédét ${ }^{13}$ dans lequel la structure changée de l'indirect ordinaire est due, croyons-nous, aux facteurs psychologiques (affectifs, impulsifs):

Quant il (sc. Guigemar) a la parole oïe, Ducement la dame mercie:

od li sujurnerat, ceo dit.

(Marie de France, Guigemer, v. 359-61)

Par sez serjans lez fet tost prendre.

Ne les fera pas, ce dit, pendre, Mes escorchier vif et saler.

Tantos lez a fet avaler

En la plus fort chartre qu'il ait.

(Galeran de Bretagne, v. 4989-93)

${ }^{13}$ Ce n'est qu'exceptionnellement qu'on trouve l'incise dit il dans notre tournure: Puis s'arestut e esgarda, Lur dous beautez uit e mira: Unques meis, dist il, ceo li. semble, Ne uit si bele gent ensemble. (Rou, v. 549-552). 
Comença mei a congeër

Mout laidement de son païs:

$J a$ ne sereit, ço dist, amis

$A$ ceus de Troie nul jor mais,

N'o eus n'avreit triue ne pais...

(Le Roman de Troie, v. 3598-3602)

Il (sc. Aligrés) prent une lance pleniere,

Grosse et forte de grant maniere,

De Percheval s'est eslongiez:

Ja mais ses doels n'ert elegiés,

Ce dist, s'il n'abat Percheval.

(Gerb. de Motr., Perceval, v, 8017-21)

Atant s'est a la terre assis

$E$ dit que tel mal l'en ad pris

Qu'il ne puet mes sur piez ester,

Le quor, ço dit; li deit crever.

(Gui de Warewic, v, 4241-44)

Dist li paiens: "Or me faites entendre.

Aumons vos mande . . . . . . . . . . .

Qu'en sa merchi vos alés trestolt rendre.

Rien ne valroit envers lui li deffendre,

Toltes vos armes li venés molt tost rendre.

Ne vos volra, cho dist, de plus raiendre,

Ne mais le col desos l'espee tenare

Et puis apriés vos en porés descendre;"

( La Chanson d'Aspremont, v. 3129-31)

Coarz en fu si esperduz

Que onques puis ne fu velis.

Del signe qu'ot veî s'esmaie.

Lors s'est muchez en une haie:

D'iloc, ce dit, esgardera

Quel justice l'en en fera.

(Le Roman de Renart, I, v. 1365-70)

...et pourchacierent au patriarche de Jherusalem qu'il feroit laissier le roiaume le roi Guion, car il n'estoit mie dignes, ce disoient, d'estre rois.

(Ménestrel, § 29), etc., etc. (14)

Mais, le pas suivant à faire pour aboutir à la forme finale et véritable - telle que nous la connaissons surtout chez les auteurs après 1850 - du style indirect libre, c'est l'omission, l'ellipse de l'incise: le conteur s'identifiant complètement à son personnage oublie, en quelque sorte, le vrai rapporteur des faits, il laisse au lecteur de le deviner lui-meme, ce qui très souvent- nous l'avons déjà vu - ne va pas sans prêter à l'ambiguïté.

On aura remarqué que, parmi les exemples que nous venons de donner, il y a aussi des cas comportant le verbe au futur: or, plusieurs de ces cas, avec l'incise supprimée, feraient à juste titre penser aux exemples au futurum 
advocaticum mentionnés plus haut; à notre avis, ces cas-là font plus que seulement suggérer l'idée d'une sorte de l'indirect libre, car, comptant avec l'ellipśe de l'incise ce dit, etc. qui, selon nous, s'y était effectuée, on n'aurait indubitablement point tort d'y voir des formes véritables du style indirect libre.

Acceptant notre explication de l'origine du style indirect libre en ancien français qui serait due à l'éllipse de l'incise ce dit, etc., on admet en même tcmps aussi non seulement que cette incise peut être intercalée dans n'importe quel exemple de l'indirect libre, mais aussi que cette incise pourrait nous aider à éclaircir maints passages équivoques dans la vieille langue: si l'incise peut y être intercalée en s'y adaptant bien, on peut presque avec certitude accepter la présence du style indirect libre, sinon on a affaire au récit de l'auteưr lui-même. Voici quelques exemples, qui, croyons-nous, admettent cette possibilité (l'incise y est intercalée par nous!) ${ }^{14}$

Li emperere par sa grant poestet

VII. anz tuz plens ad en Espaigne estet;

Prent i chastels e alquantes citez.

Li reis Marsilie s'en purcacet asez:

Al premer an fist ses brefs seieler,

Fn Babilonie Baligant ad mandet, Co est l'amirail, le viel d'antiquitet, Tut survesquiet e Virgilie e Omer;

En Sarraguce alt sucurre li ber

$E$; s'il nel fait, (ço dist), il guerpirat ses deus

E tuz ses ydeles que il soelt adorer,

Si recevrat seinte chrestientet,

A Charlemagne se vuldrat acorder.

E cil est loinz, si ad mult demuret;

(La Chanson de Roland, v. 2609-22)

(En effet, Bédier traduit: s'il (sc. Baligant) ne le fait, Marsile reniera ses. dieux..., c'est-à-dire les vers en question reproduisent les paroles du personnage (sc. de Marsile), et non pas le récit du conteur.)

Eliduc li (sc. à sa femme) ad otrié.

$\mathrm{E}$ bonement cungé doné:

Tute sa volunté fera (ceo dist)

$E$ de sa tere li durra.

Pres del chastel ...

Ea ad fet fere sun muster,

$\mathrm{E}$ ses meisuns edifier.

(Marie de France, Eliduc, v. 1132-38)

Quant (Gui) vit gesir sanglant le ber, S'il nel venge (ço dist), ja n'iert haité;

Pur lui grant duel ad demené.

(Gui de Warewic; v. 4791-93)

${ }^{14}$ Comp. aussi les exemplés douteux donnés plus haut et considérés, par nous comme des cas de l'indirect libre. 
Gimbert li taissons se leva; se il puet, (ço dist), Renart aidera, que ses cousins germains estoit.

(Le Roman de Renart, I, v. 215-17)

Et la dame se rapensa

Qu'ele avoit mout grant tort eii.

Mout vousist (co dist) bien avoir seü

Coment ele (sc. sa servante) porroit prover

Qu'on porroit chevalier trover

Meillor qu'onques ne fu ses sire.

Mout volentiers li orroit dire,

Mais ele li a defendu.

En cest voloir a atendu

Jusqu'a tant que cele revint.

(Chrétien de Tr., Le Chevalier au lion, v, 249-58)

(L'emploi du conditionnel présent, lui aussi, parle en faveur de l'indirect libre, l'auteur, de son point de vue, aurait dû faire usage du conditionnel passé. G. Paris et E. Langlois, Chrestomathie du moyen âge, ont raison de traduire: Elle voudrait bien savoir... Bien volontiers elle le lui entendrait dire...)

Peut-être n'aurions-nous point tort d'admettre la même possibilité aussi pour les complétives plus distancées dépendant du verbe principal jurer (v. plus haut) et dans lesquelles la conjonction que est omise, p. ex.

Puis me fist sur seinz jurer

Qu'en cest message deusșe aler

$\mathrm{E}$ a lui puis repairer;

Se nul de ces ne peusse amener,

A. grant dolur, (ço dist), me freit morir,

Nule rien ne m'en purreit garir,

Ensemble od mei mes quinze fiz;

(Gui de Warewic, v. 8125-31), etc., etc.

L'incise énonciative ce dit, caractéristique pour le style indirect libre en ancien français, a bientôt pris la forme dit-il, s'identifiant ainsi à l'incise du style diréct. Elle apparaît encore très souvent dans l'indirect libre tout jusqu'à l'époque moderne, surtout là où l'auteur craint la confusion du style indirect libre avec la simple narration. Comp.:

La lice lui demande encore une quinzaine.

Ses petits ne marchoient, disoit-elle, qu'à peine;

(La Fontaine, Fables, II, .7)

Comment excuser une si grande imprudence, et qu'était devenue l'extrême discrétion de ce prince, dont elle avoit été si touchée? Il a été discret, disoitelle, tant qu'il a cru être malheureux.

(Mme de Lafayette, La Princesse de Chlèves, 3e partie) 
Averti par sa mauvaise humeur, je lui (sc. au comte) proposais une partie; alors il coquetait: — D'abord il était trop tard, disait-il, puis je ne m'en souciais pas.

(Balzac, Le lys dans la vallée, p. 65)

Il réitéra sa demande que la bonne femme se refusa nettement à satisfaire .Elle ne voulait pas, disait-elle, enlever la crème des potées de lait destinées à faire le beurre.

(Id., Le médecin de campagne, ch. 3)

J'ai rencontré une fois un agent du F. B. I. Comme j'étais moi-même fonctionnaire en mission, nous échangeâmes d'abord des propos d'ordre professionnel - indemnités de déplacement, régimes des retraites, - puis j'orientai la conversation vers le maccarthysme. Mon interlocuteur en parlait avec le mépris glacial du médecin pour le rebouteux. Ces gens-là, disait-il, r'étaient que des bousilleurs, des piétineurs de plates-bandes, sans délicatesse ni discernement. Je lui demandai...

(R. Escarpit, Les deux font la paire, p. 239; Paris 1959), etc. (15)

L'indirect à incise est aussi couramment pratiqué par Marmontel qui cependant recourt, pour varier son style, aussi à l'indirect sans incise. Il n'est pas sans intérêt pour nous de noter ce qu'il dit lui-même à ce sujet: "Je proposai, il y a quelques années, dans l'un des articles de l'Encyclopédie, de supprimer les dit-il et les dit-elle du dialogue vif et pressé. J'en ai fait l'essai dans ces Contes; et il me semble qu'il a réussi. Cette manière de rendre le récit plus rapide n'est pénible qu'au premier instant: dès qu'on y est accoutumé, il fait briller le talent de bien lire.i (Préface; cité d'après $\mathbf{M}$. Lipps, o. c., p. $163 \mathrm{~s}$ ). Voici un exemple de l'application de ce principe:

La belle prude, suivant l'usage, opposait toujours quelque faible résistance aux désirs d'Alcibiade. C'était une chose épouvantable! elle ne pouvait y penser sans rougir. Il fallait aimer comme elle aimait, pour s'y résoudre. Elle aurait voulu pour tout au monde qu'il fût moins empressé.. Alcibiade la prit au mot.

(Contes, Alcibiade, p. 2)

${ }^{15}$ Comp. aussi pour l'anglais: Mrs. Jervis says, he asked her if I kept the men at a distance; for, he said, I was very pretty; and to be drawn in to have any of them might be my ruin, and make me poor and miserable betimes. (Richardson, Pamela, I, Letter 6); But my mother... would not consent to take a fraction more than was due to her, and was obstinately unwilling to be content with less. It was not yet seven, she said, by a long way; she knew her rights and she would have them; and she was still arguing with, when a little low whistle sounded. (R. C. Stevenson, Treasure Island, Ch. IV), etc.

Voici aussi ce que dit Walzel, $l$. c., pour l'écrivain allemand Wieland: "Wieland sucht sie (sc. die erlebte Rede) noch zu rechtfertigen durch ein eingeschobenes und eingeklammertes »dacht'er《. Dann wird bezeichnendes Merkmal gerade das Fehlen solcher Einfuihrung. Gewiss kam das der zunehmenden Neigung entgegen, Rede, und Gegenrede ohne Angabe eines "sagte er" oder wantwortete sie zu bringen." Walzel aussi, donc, compte avec l'ellipse de l'incise. 
Averti par sa mauvaise humeur, je lui (sc. au comte) proposais une partie; alors il coquetait: - D'abord il était trop tard, disait-il, puis je ne m'en souciais pas.

(Balzac, Le lys dans la vallée, p. 65)

Il réitéra sa demande que la bonne femme se refusa nettement à satisfaire .Elle ne voulait pas, disait-elle, enlever la crème des potées de lait destinées à faire le beurre.

(Id., Le médecin de campagne, ch. 3)

J'ai rencontré une fois un agent du F. B. I. Comme j'étais moi-même fonctionnaire en mission, nous échangeâmes d'abord des propos d'ordre professionnel - indemnités de déplacement, régimes des retraites, - puis j'orientai la conversation vers le maccarthysme. Mon interlocuteur en parlait avec le mépris glacial du médecin pour le rebouteux. Ces gens-là, disait-il, n'étaient que des bousilleurs, des piétineurs de plates-bandes, sans délicatesse ni discernement. Je lui demandai...

(R. Escarpit, Les deux font la paire, p. 239; Paris 1959), etc. (15)

L'indirect à incise est aussi couramment pratiqué par Marmontel qui cependant recourt, pour varier son style, aussi à l'indirect sans incise. Il n'est pas sans intérêt pour nous đe noter ce qu'il dit lui-même à ce sujet: "Je proposai, il y a quelques années, dans l'un des articles de l'Encyclopédie, de supprimer les dit-il et les dit-elle du dialogue vif et pressé. J'en ai fait l'essai dans ces Contes; et il me semble qu'il a réussi. Cette manière de rendre le récit plus rapide n'est pénible qu'au premier instant: dès qu'on y est accoutumé, il fait briller le talent de bien lire.) (Préface; cité d'après M. Lipps, o. c., p. $163 \mathrm{~s}$ ). Voici un exemple de l'application de ce principe:

La belle prude, suivant l'usage, opposait toujours quelque faible résistance aux désirs d'Alcibiade. C'était une chose épouvantable! elle ne pouvait y penser sans rougir. Il fallait aimer comme elle aimait, pour s'y résoudre. Elle aurait voulu pour tout au monde qu'il fût moins empressé.. Alcibiade la prit au mot.

(Contes, Alcibiade, p. 2)

${ }^{15}$ Comp. aussi pour l'anglais: Mrs. Jervis says, he asked her if I kept the men at a distance; for, he said, I was very pretty; and to be drawn in to have any of them might be my ruin, and make me poor and miserable betimes. (Richardson, Pamela, I, Letter 6); But my mother... would not consent to take a fraction more than was due to her, and was obstinately unwilling to be content with less. It was not yet seven, she said, by a long way; she knew her rights and she would have them; and she was still arguing with, when a little low whistle sounded. (R. C. Stevenson, Treasure Island, Ch. IV), etc.

Voici aussi ce que dit Walzel, $l$. c., pour l'écrivain allemand Wieland: "Wieland sucht sie (sc. die erlebte Rede) noch zu rechtfertigen durch ein eingeschobenes und eingeklammertes "dacht'er«. Dann wird bezeichnendes Merkmal gerade das Fehlen solcher Einführung. Gewiss kam das der zunehmenden Neigung entgegen, Rede, und Gegenrede ohne Angabe eines "sagte er" oder vantwortete sie» zu bringen." Walzel aussi, donc, compte avec l'ellipse de l'incise. 
angleščino pa tudi avtor te razprave navaja nekaj primerov naše konstrukcije iz precej starejše dobe.

Avtor.skuša tudi dokazati, da je bil naš stilistični postopek že v stari francoščni mnogo pogostejši, kot to dajo sklepati izvajanja $M$. Lippsove in $G$. Lerchove.

Končno se avtor dotakne tudi problema o izvoru tega pojava; skuša ga najti v zelo pogostni starofrancoski obliki odvisnega govora, ki tvori nekak most med navadnim odvisnim ter prostim odvisnim govorom, namreč:

Fremaus presentent a chascun;

Me sire Gavains en prist un,

Si l'a tantost a son col mis,

Mais Tristrans n'a pas le sien pris:

Fremail, ce dist, ne meteroit,

$\mathrm{Ne}$ en son doit anel ' $n$ 'aroit

Dusqu'al terme qu'il avoit mis

$A$ tele $a$ en alcun pais.

(Gerb. de Motr., Perceval, v. 3671-78)

To obliko sámo izvaja avtor iz navadnega odvisnega govora, tudi pogostnega v starem jeziku:

Ço dist lị reis que sa guere out finee

( La Chanson de Roland, v. 705)

Iz vzrokov afektivne narave, tj. pod vtisom dogodkov, povedanih $\mathrm{v}$ odvisniku, avtor impulzivno najprej poroča o njih in šele naknadno $z$ vrinjenim stavkom ço dist (ki je le preneseni verbum dicendi glavnega stavka) odkrije pravega pripovedovalca teh dogodkov.

Nadaljnji korak $\mathrm{k}$ popolni obliki naše stilistične posebnosti pa je narejen $z$ izpustitvijo vrinjenega stavka ce dist, za kar navaja avtor nekatere možnosti že v starem jeziku. Vendar se vrinjeni ce dist (oziroma dit-il $\mathrm{v}$ modernizirani obliki) najde vse do najnovejše dobe, zlasti tam, kjer se hoče avtor izogniti nejasnosti. Podrobnosti in številne primere gl. $v$ francoskem delu razprave. 